\title{
A Class of Generalized Mixed Variational-Hemivariational Inequalities I: Existence and Uniqueness Results *
}

\author{
Yunru Bai $\lesseqgtr$ Stanisław Migórski ${ }^{\ddagger}$ and Shengda Zeng ${ }^{\S}$
}

\begin{abstract}
We investigate a generalized Lagrange multiplier system in a Banach space, called a mixed variational-hemivariational inequality (MVHVI, for short), which contains a hemivariational inequality and a variational inequality. First, we employ the Minty technique and a monotonicity argument to establish an equivalence theorem, which provides three different equivalent formulations of the inequality problem. Without compactness for one of operators in the problem, a general existence theorem for (MVHVI) is proved by using the Fan-Knaster-Kuratowski-Mazurkiewicz principle combined with methods of nonsmooth analysis. Furthermore, we demonstrate several crucial properties of the solution set to (MVHVI) which include boundedness, convexity, weak closedness, and continuity. Finally, a uniqueness result with respect to the first component of the solution for the inequality problem is proved by using the Ladyzhenskaya-Babuška-Brezzi (LBB) condition. All results are obtained in a general functional framework in reflexive Banach spaces.
\end{abstract}

Key words. Mixed variational-hemivariational inequality, Fan-Knaster-KuratowskiMazurkiewicz principle, Ladyzhenskaya-Babuška-Brezzi (LBB) condition, upper semicontinuity, existence, uniqueness.

2010 Mathematics Subject Classification. 35J50, 35J88, 35M87, 74H20, 74H25.

* This project has received funding from the European Union's Horizon 2020 Research and Innovation Programme under the Marie Skłodowska-Curie grant agreement No. 823731 - CONMECH. It is supported by the National Science Center of Poland under Maestro Project No. UMO-2012/06/A/ST1/00262, and National Science Center of Poland under Preludium Project No. 2017/25/N/ST1/00611. The second author is also supported by the Natural Sciences Foundation of Guangxi Grant No. 2018JJA110006, the Beibu Gulf University Project No. 2018KYQD03, and the Project Financed by the Ministry of Science and Higher Education of Republic of Poland under Grant No. 4004/GGPJII/H2020/2018/0.

$\dagger$ Jagiellonian University in Krakow, Faculty of Mathematics and Computer Science, ul. Lojasiewicza 6, 30348 Krakow, Poland. E-mail address: yunrubai@163.com.

$¥$ College of Sciences, Beibu Gulf University, Qinzhou, Guangxi 535000, P.R. China, and Jagiellonian University in Krakow, Chair of Optimization and Control, ul. Lojasiewicza 6, 30348 Krakow, Poland. Tel.: +48-12-6646666. E-mail address: stanislaw.migorski@uj.edu.pl.

$\S$ Jagiellonian University in Krakow, Faculty of Mathematics and Computer Science, ul. Lojasiewicza 6, 30348 Krakow, Poland. Corresponding author. Tel.: +86-18059034172. E-mail address: shengdazeng@gmail.com; shdzeng@hotmail.com; zengshengda@163.com. 


\section{Introduction and problem statement}

In many complicated physical processes and engineering applications, mathematical models based on variational inequality formulations and their generalizations play an important role. Recently, a new class of systems with Lagrange multipliers which consists of a variational inequality has drawn a great attention. The reason is that such systems are a powerful mathematical tool to model and solve a variety of problems in engineering areas such as dynamic vehicle routing problems, contact problems in mechanics, the behavior of Navier-Stokes fluids, the penetration phenomenon of magnetic field, etc.

The most representative recent results in this area are the following: CojocaruMatei [5] who have discussed the unique solvability for a class of frictional contact problems governed by the $p$-Laplace operator, which can be formulated as a mixed variational inequality; Matei et al. [19] have employed the Lagrange multipliers method to consider a deformable body in frictionless unilateral contact with a moving rigid obstacle, and explored an efficient algorithm approximating the weak solution for a more general case of a two-body contact problem including friction; Han-Reddy [10] who have analyzed the finite element method for a class of mixed variational inequalities of the second kind which arises in elastoplastic problems; Sofonea-Matei [32] who have considered a new class of mixed variational problems, and proved existence, uniqueness as well as continuous dependence results by applying generalized saddle point formulations and various estimates, combined with a fixed point argument. We refer the reader to [13, 14, 15, 16, 17, 18, 19, 20, 30, 31] and the references therein for a more detailed discussion of this topic.

On the other hand, the notion of a hemivariational inequality was first introduced and studied by P.D. Panagiotopoulos [27, 28, 29] in the early 1980s who used this mathematical tool to describe and solve complicated problems modeling various physical phenomena. After that, more and more researchers are attracted to boost the development of the theory and applications of hemivariational inequalities, since they can be applied to a wide range of engineering problems involving nonmonotone and possibly multivalued constitutive and interface laws for deformable bodies, see e.g. [1, 2, 13, 11, 12, 22, 21, 23, 24, 25, 26, 33]. Very recently, Matei [17] has studied an abstract system with Lagrange multipliers, called a mixed variational-hemivariational inequality, which consists of a hemivariational inequality and a variational inequality, and then demonstrated three existence theorems which are illustrated by two applications. However, in paper [17], some problems concerning mixed variationalhemivariational inequalities, such as uniqueness, are left open. Based on this motivation, in this paper, we will develop a new class of abstract mixed variationalhemivariational inequalities in a general functional framework.

Let $V$ and $E$ be reflexive Banach spaces, and $\Lambda$ be a nonempty subset of $E$. We denote by $\langle\cdot, \cdot\rangle$ the duality pairing between $V$ and its dual $V^{*}$. Let $X$ be another Banach space. Given an operator $A: V \rightarrow V^{*}$, a function $J: X \rightarrow \mathbb{R}$, a bilinear function $b: V \times E \rightarrow \mathbb{R}$, an operator $\gamma: V \rightarrow X$ and an element $f \in V^{*}$, the purpose of this paper is to study the following abstract generalized mixed variational-hemivariational inequality. 
Problem 1. Find $(u, \lambda) \in V \times \Lambda$ such that the following two inequalities hold

$$
\begin{aligned}
& \langle A(u), v-u\rangle+b(v-u, \lambda)+J^{0}(\gamma u ; \gamma v-\gamma u) \geq\langle f, v-u\rangle \text { for all } v \in V, \\
& b(u, \rho-\lambda) \leq 0 \text { for all } \rho \in \Lambda .
\end{aligned}
$$

To highlight the motivation to study Problem 1, we mention below its particular cases.

(i) Let $j: \mathbb{R}^{d} \rightarrow \mathbb{R}$ be a Lipschitz continuous function and $\Omega \subset \mathbb{R}^{d}(d \geq 2)$ be a bounded domain with smooth boundary $\Gamma=\partial \Omega$. If $\gamma: V \rightarrow L^{p}\left(\Gamma ; \mathbb{R}^{r}\right)$ is a linear, bounded and compact operator with $2 \leq p<\infty$ and $r \geq 1$, and $J$ is defined by

$$
J(v)=\int_{\Gamma_{1}} j(\gamma v(x)) d \Gamma \quad \text { for all } v \in V,
$$

where $\Gamma_{1} \subset \Gamma$ is such that meas $\left(\Gamma_{1}\right)>0$, then Problem 1 reduces to

$$
\begin{aligned}
& \langle A(u), v-u\rangle+b(v-u, \lambda)+\int_{\Gamma_{1}} j^{0}(u(x) ; v(x)-u(x)) d \Gamma \geq\langle f, v-u\rangle \text { for all } v \in V, \\
& b(u, \rho-\lambda) \leq 0 \text { for all } \rho \in \Lambda,
\end{aligned}
$$

which has been recently studied by Matei [17.

(ii) If $J \equiv 0$, then Problem 1 becomes

$$
\left\{\begin{array}{l}
\langle A(u), v\rangle+b(v, \lambda)=\langle f, v\rangle \text { for all } v \in V \\
b(u, \rho-\lambda) \leq 0 \text { for all } \rho \in \Lambda .
\end{array}\right.
$$

This mixed variational inequality has been investigated by Cojocaru-Matei [5].

The aim of this paper is to extend the theoretical results from [17] to a generalized mixed variational-hemivariational inequality in a general functional framework, Problem 1, and provide positive answers to open problems remained in [17]. The main novelties of the paper are described as follows.

First, in the study of Problem 1, we do not require that function $J$ is Lipschitz continuous and the operator $\gamma: V \rightarrow X$ is compact. This extends the scope of applications for mixed variational-hemivariational inequality. Besides, the main core of the proof is completely different from the one carried out in [17, here we employ the well-known Fan-Knaster-Kuratowski-Mazurkiewicz theorem, not a fixed point principle.

Second, our results can be applied to a special case of Problem 1 in which $b \equiv 0$, and the problem reduces to the following "pure" hemivariational inequality

$$
\langle A(u), v-u\rangle+J^{0}(\gamma u ; \gamma v-\gamma u) \geq\langle f, v-u\rangle \text { for all } v \in V .
$$

In fact, the above inequality has been explored by many scholars from the mathematical and application points of view under the crucial hypothesis that operator $\gamma$ is compact, see e.g. [6, 34, 35]. However, in our results, we will overcome this assumption. 
Third, for the first time, we provide the uniqueness theorem to Problem 1 with respect to the first component $u \in V$ of solution. In the meanwhile, we develop several important properties of the solution set to Problem 1, which include boundedness, convexity, weak closedness, continuity, etc. We believe that those results will be found useful in a number of complex problems involving a mixed variational-hemivariational inequality as a subsystem, for instance, in optimal control problems driven by mixed variational-hemivariational inequalities.

The outline of the paper is as follows. Basic notation and preliminary material needed in the sequel are recalled in Section 2, In Section 3, we deliver our main results concerning Problem 1 which include a Minty type equivalence result, a general existence theorem, several significant properties of the solution set, and a uniqueness result.

\section{Background material}

In this section, we briefly review basic notation and some results which are needed in the sequel. For more details, we refer to monographs [4, 7, 8, 36].

Throughout the paper, we denote by $\langle\cdot, \cdot\rangle_{Y^{*} \times Y}$ the duality pairing between a Banach space $Y$ and its dual $Y^{*}$. The norm in a normed space $Y$ is denoted by $\|\cdot\|_{Y}$. Given a subset $D$ of $Y$, we write $\|D\|_{Y}=\sup \left\{\|v\|_{Y} \mid v \in Y\right\}$. If no confusion arises, we often drop the subscripts. Besides, we denote by $\mathcal{L}\left(Y_{1}, Y_{2}\right)$ the space of linear and bounded operators from a normed space $Y_{1}$ to a normed space $Y_{2}$ endowed with the usual norm $\|\cdot\|_{\mathcal{L}\left(Y_{1}, Y_{2}\right)}$.

We begin with definitions and properties of semicontinuous multivalued mappings.

Definition 2. Let $X$ and $Y$ be topological spaces, and $F: X \rightarrow 2^{Y}$ be a multivalued mapping. We say that $F$ is

(i) upper semicontinuous (u.s.c., for short) at $x \in X$ if, for every open set $O \subset Y$ with $F(x) \subset O$ there exists a neighborhood $N(x)$ of $x$ such that $F(N(x)):=$ $\cup_{y \in N(x)} F(y) \subset O$. If this holds for every $x \in X$, then $F$ is called upper semicontinuous.

(ii) closed at $x_{0} \in X$, if for every sequence $\left\{\left(x_{n}, y_{n}\right)\right\} \subset G r(F)$ such that $\left(x_{n}, y_{n}\right) \rightarrow$ $\left(x_{0}, y_{0}\right)$ in $X \times Y$, we have $\left(x_{0}, y_{0}\right) \in G r(F)$, where $G r(F)$ is the graph of the multivalued mapping $F$ defined by

$$
G r(F):=\{(x, y) \in X \times Y \mid y \in F(x)\} .
$$

We say that $F$ is closed (or $F$ has a closed graph), if it is closed at every $x_{0} \in X$.

The following theorem gives a criterium for upper semicontinuity.

Proposition 3. [22, Proposition 3.8] Let $X$ and $Y$ be two topological spaces, and $F: X \rightarrow 2^{Y}$. The following statements are equivalent:

(i) $F$ is u.s.c.. 
(ii) for every closed set $C \subset Y$, the set

$$
F^{-}(C):=\{x \in X \mid F(x) \cap C \neq \emptyset\}
$$

is closed in $X$.

Theorem 4. [7, Proposition 4.1.9] Let $X$ be a topological space, $Y$ be a regular topological space, and $F: X \rightarrow 2^{Y}$ be an upper semicontinuous multivalued mapping with closed values. Then $F$ is closed.

Let $\left(V,\|\cdot\|_{V}\right)$ be a Banach space. A function $J: V \rightarrow \mathbb{R}$ is called to be locally Lipschitz continuous at $u \in V$, if there exist a neighborhood $N(u)$ of $u$ and a constant $L_{u}>0$ such that

$$
|J(w)-J(v)| \leq L_{u}\|w-v\|_{V} \text { for all } w, v \in N(u) .
$$

Definition 5. Given a locally Lipschitz function $J: V \rightarrow \mathbb{R}$, we denote by $J^{0}(u ; v)$ the generalized (Clarke) directional derivative of $J$ at the point $u \in V$ in the direction $v \in V$ defined by

$$
J^{0}(u ; v)=\limsup _{\lambda \rightarrow 0^{+}, w \rightarrow u} \frac{J(w+\lambda v)-J(w)}{\lambda} .
$$

The generalized gradient of $J: V \rightarrow \mathbb{R}$ at $u \in V$ is given by

$$
\partial J(u)=\left\{\xi \in V^{*} \mid J^{0}(u ; v) \geq\langle\xi, v\rangle \text { for all } v \in V\right\} .
$$

The generalized gradient and generalized directional derivative of a locally Lipschitz function enjoy many nice properties and rich calculus. Here we just collect below some basic and crucial results, see e.g. [22, Proposition 3.23].

Proposition 6. Let $J: V \rightarrow \mathbb{R}$ be a locally Lipschitz continuous function. Then

(i) for each $u \in V$, the function $V \ni v \mapsto J^{0}(u ; v) \in \mathbb{R}$ is positively homogeneous, subadditive, and satisfies $\left|J^{0}(u ; v)\right| \leq L_{u}\|v\|_{V}$ for all $v \in V$, where $L_{u}>0$ is the Lipschitz constant of $J$ near $u$.

(ii) the function $V \times V \ni(u, v) \mapsto J^{0}(u ; v) \in \mathbb{R}$ is upper semicontinuous.

(iii) for each $v \in V$, we have $J^{0}(u ; v)=\max \left\{\left\langle u^{*}, v\right\rangle \mid u^{*} \in \partial J(u)\right\}$.

We conclude this section with the following Fan-Knaster-Kuratowski-Mazurkiewicz theorem (F-KKM theorem, for short) which will play an important role in the proof of existence of solutions to the inequality problems in Section 3. Its proof can be found in Ky Fan [9].

THEOREM 7. [9] Let $K$ be a nonempty subset of a Hausdorff topological vector space $E$ and $G: K \rightarrow 2^{E}$ be a multivalued mapping with the following properties:

(a) $G$ is a KKM mapping, that is, for any $\left\{v_{1}, v_{2}, \ldots, v_{N}\right\} \subset K$, one has that its convex hull co $\left\{v_{1}, v_{2}, \ldots, v_{n}\right\}$ is contained in $\bigcup_{i=1}^{n} G\left(v_{i}\right)$,

(b) for every $v \in K, G(v)$ is closed in $E$,

(c) for some $v_{0} \in K, G\left(v_{0}\right)$ is compact in $E$.

Then, we have $\bigcap_{v \in K} G(v) \neq \emptyset$. 


\section{$3 \quad$ Existence and uniqueness results}

The section is devoted to deliver the main results of this paper, which contain five theorems and two corollaries. More precisely, the first theorem, Theorem 9, provides three various equivalent formulations for Problem 1 by using the Minty approach and a monotone argument. In the second theorem, Theorem 10, we employ the Fan-KnasterKuratowski-Mazurkiewicz theorem and the theory of nonsmooth analysis to establish an existence result to Problem 1, in which we do not require that the operator $\gamma$ is compact. Next result, Theorem 11 is devoted to explore some important properties of solution set of Problem 1, which include boundedness, convexity, weak closedness, and continuity. Subsequently, a uniqueness theorem, Theorem 15, for Problem 1 is established by using the Ladyzhenskaya-Babuška-Brezzi (LBB) condition. The last result, Theorem 16. presents a continuity result (or stability result) for the solution mapping.

To establish main results on Problem 1, we now impose the following assumptions on its data. Let $\left(V,\|\cdot\|_{V}\right)$ and $\left(E,\|\cdot\|_{E}\right)$ be two reflexive Banach spaces.

$H(h): h: V \rightarrow \mathbb{R}$ is such that $h\left(0_{V}\right)=0$ and

(i) $\lim \sup _{t \rightarrow 0^{+}} \frac{h(t v)}{t} \geq 0$ for all $v \in V$.

(ii) for all $\left\{v_{n}\right\} \subset V$ with $v_{n} \rightarrow v$ weakly in $V$, we have $h(v) \leq \limsup _{n \rightarrow \infty} h\left(v_{n}\right)$.

(iii) for all $v \in V \backslash\left\{0_{V}\right\}$, we have $h(v)>0$.

$H(J): J: X \rightarrow \mathbb{R}$ is such that

(i) $J$ is locally Lipschitz continuous.

(ii) there exist $\theta \geq 0, \alpha_{J} \geq 0$ and $\beta_{J}>0$ such that

$$
J^{0}(v ;-v) \leq \alpha_{J}+\beta_{J}\|v\|_{X}^{\theta} \quad \text { for all } v \in X .
$$

(iii) the multivalued mapping $X \ni v \mapsto \partial J(v) \subset X^{*}$ is bounded, i.e., $\partial J$ maps bounded subsets of $X$ into bounded subsets of $X^{*}$.

$H(A): A: V \rightarrow V^{*}$ is such that

(i) for any $w, v \in V$ fixed, it holds

$$
\limsup _{t \rightarrow 0^{+}}\langle A(t w+(1-t) v), w-v\rangle \leq\langle A(v), w-v\rangle .
$$

(ii) the mapping $A \cdot+\gamma^{*} \partial J(\gamma \cdot): V \rightarrow 2^{V^{*}}$ is $h$-relaxed monotone on $V$, i.e.,

$$
\left\langle A(u)+\gamma^{*} \xi_{u}-A(v)-\gamma^{*} \xi_{v}, u-v\right\rangle \geq h(u-v)
$$

for all $\xi_{u} \in \partial J(\gamma u), \xi_{v} \in \partial J(\gamma v)$ and $u, v \in V$.

(iii) $A$ is coercive in the following sense

$$
\lim _{v \in V,\|v\|_{V} \rightarrow+\infty} \frac{\langle A v, v\rangle}{\|v\|_{V}^{\max \{\theta, 1\}}}=+\infty
$$


where $\theta \geq 0$ is given in hypothesis $H(J)($ ii).

(iv) $A$ is a bounded operator.

$H(b)$ : The bilinear function $b: V \times E \rightarrow \mathbb{R}$ is bounded and satisfies the following inequality

$$
\inf _{\rho \in E \backslash\left\{0_{E}\right\}} \sup _{v \in V \backslash\left\{0_{V}\right\}} \frac{b(v, \rho)}{\|v\|_{V}\|\rho\|_{E}} \geq \alpha_{b}
$$

for some $\alpha_{b}>0$.

$\underline{H(\gamma)}: \gamma: V \rightarrow X$ is a linear and continuous operator.

In the following we comment on the above hypotheses.

REMARK 8. Various kinds of monotonicity of operator $A \cdot+\gamma^{*} \partial J(\gamma \cdot)$ can be obtained by choosing a suitable function $h$. It is worth to mention that if hypotheses $H(h)(i)$ and (iii) are specified by $h(t v)=t^{\tau} h(v)$ for all $v \in V$ and $t>0$ with $\tau>1$, and $h(v) \geq c_{h}\|v\|_{V}^{q}$ for all $v \in V$ with some $c_{h}>0$ and $q>1$, respectively, then hypotheses $H(h)$ reduces to the one considered by Cojocaru-Matei [5]. In particular, function $h(v)=m_{A}\|v\|^{\tau}$ for $v \in V$ with $m_{A}>0$ and $\tau \geq 1$ enjoys $H(h)$, and then $H(A)(i i)$ means that $A \cdot+\gamma^{*} \partial J(\gamma \cdot)$ is $\tau$-strongly monotone. Note also that hypothesis $H(A)(i)$ is weaker then the hemicontinuity of operator A, see [22, Definition 3.68].

Let us turn to the hypotheses $H(J)$. If the generalized gradient $\partial J$ has a sublinear growth, namely,

$$
\|\partial J(v)\|_{X^{*}} \leq c_{J}+d_{J}\|v\|_{X} \text { for all } v \in X
$$

for some $c_{J} \geq 0$ and $d_{J}>0$, then hypothesis $H(J)$ (iii) is clearly satisfied.

The inequality (3) is usually called the Ladyzhenskaya-Babuška-Brezzi (LBB) condition which widely appears in the literature.

The first result of the paper provides three different equivalent formulations of Problem 1 by applying the Minty approach, in which $V$ and $\Lambda$ are replaced by the nonempty, closed and convex subsets of $V$ and $E$, respectively.

TheOREM 9. Let $K$ and $Y$ be nonempty, closed and convex subsets of $V$ and $E$, respectively. Assume that hypotheses $H(A)(i)-(i i), H(J)(i), H(h)(i)$, and $H(\gamma)$ hold. If $b: V \times E \rightarrow \mathbb{R}$ is a bilinear and bounded function, then $(u, \lambda) \in K \times Y$ is a solution to the following mixed variational-hemivariational inequality

$$
\begin{aligned}
& \langle A(u), v-u\rangle+b(v-u, \lambda)+J^{0}(\gamma u ; \gamma v-\gamma u) \geq\langle f, v-u\rangle \text { for all } v \in K \\
& b(u, \rho-\lambda) \leq 0 \quad \text { for all } \rho \in Y
\end{aligned}
$$

if and only if it solves one of the following problems

(i) $(u, \lambda) \in K \times Y$ is such that

$$
\begin{gathered}
\langle A v, v-u\rangle+b(v-u, \lambda)+J^{0}(\gamma v ; \gamma v-\gamma u) \\
\geq\langle f, v-u\rangle+h(v-u) \text { for all } v \in K, \\
b(u, \rho-\lambda) \leq 0 \text { for all } \rho \in Y .
\end{gathered}
$$


(ii) $(u, \lambda) \in K \times Y$ is such that

$$
\left\{\begin{array}{r}
\langle A u, v-u\rangle+b(v, \lambda)-b(u, \rho)+J^{0}(\gamma u ; \gamma v-\gamma u) \\
\geq\langle f, v-u\rangle \text { for all } v \in K \text { and all } \rho \in Y .
\end{array}\right.
$$

(iii) $(u, \lambda) \in K \times Y$ is such that

$$
\left\{\begin{aligned}
\langle A v, v-u\rangle+b(v, \lambda)-b(u, \rho)+J^{0}(\gamma v ; \gamma v-\gamma u) \\
\geq\langle f, v-u\rangle+h(v-u) \text { for all } v \in K \text { and all } \rho \in Y .
\end{aligned}\right.
$$

Proof. (i) Let $(u, \lambda) \in K \times Y$ be a solution to problem (4) and (5). It is obvious that inequality (5) coincides with (7). Moreover, the $h$-relaxed monotonicity of operator $A \cdot+\gamma^{*} \partial J(\gamma \cdot)$ leads to

$$
\langle A v, v-u\rangle+\left\langle\xi_{v}, \gamma(v-u)\right\rangle_{X^{*} \times X} \geq\langle A u, v-u\rangle+\left\langle\xi_{u}, \gamma(v-u)\right\rangle_{X^{*} \times X}+h(v-u)
$$

for all $\xi_{u} \in \partial J(\gamma u), \xi_{v} \in \partial J(\gamma v)$ and all $u, v \in V$. Taking into account the above inequality, the property

$$
J^{0}(\gamma u ; \gamma v-\gamma u)=\max _{\xi_{u} \in \partial J(\gamma u)}\left\langle\xi_{u}, \gamma(v-u)\right\rangle_{X^{*} \times X}=\left\langle\widetilde{\xi}_{u}, \gamma(v-u)\right\rangle_{X^{*} \times X}
$$

for some $\widetilde{\xi}_{u} \in \partial J(\gamma u)$, and inequality (4), we obtain

$$
\begin{aligned}
h(v & -u)+\langle f, v-u\rangle \\
& \leq\langle A u, v-u\rangle+J^{0}(\gamma u ; \gamma v-\gamma u)+b(v-u, \lambda)+h(v-u) \\
& =\langle A u, v-u\rangle+\left\langle\widetilde{\xi}_{u}, \gamma(v-u)\right\rangle_{X^{*} \times X}+b(v-u, \lambda)+h(v-u) \\
& \leq\langle A v, v-u\rangle+\left\langle\xi_{v}, \gamma(v-u)\right\rangle_{X^{*} \times X}+b(v-u, \lambda) \quad\left(\text { for all } \xi_{v} \in \partial J(\gamma v)\right) \\
& \leq\langle A v, v-u\rangle+J^{0}(\gamma v ; \gamma v-\gamma u)+b(v-u, \lambda)
\end{aligned}
$$

for all $v \in K$. So, $(u, \lambda) \in K \times Y$ is also a solution to problem (6) and (7).

Conversely, let $(u, \lambda) \in K \times Y$ be a solution to problem (6) and (17). Then, (5) holds due to (7). It is enough to obtain (41). Let $w \in K$, and $t \in(0,1)$ be arbitrary. Taking $v=v_{t}:=t w+(1-t) u$ in (6), we employ hypotheses $H(b), H(J)(\mathrm{i}), H(\gamma)$, and Proposition 6 to get

$$
\begin{aligned}
& h(t(w-u))+t\langle f, w-u\rangle \\
& \quad \leq t\left\langle A v_{t}, w-u\right\rangle+t b(w-u, \lambda)+J^{0}(\gamma u ; t(\gamma w-\gamma u)) \\
& \quad=t\left[\left\langle A v_{t}, w-u\right\rangle+b(w-u, \lambda)+J^{0}(\gamma u ; \gamma w-\gamma u)\right],
\end{aligned}
$$

and hence

$$
\begin{aligned}
& \left\langle A v_{t}, w-u\right\rangle+b(w-u, \lambda)+J^{0}(\gamma u ; \gamma w-\gamma u) \\
& \quad \geq\langle f, w-u\rangle+\frac{h(t(w-u))}{t} .
\end{aligned}
$$


Passing to the upper limit as $t \rightarrow 0^{+}$, we now apply conditions $H(A)(\mathrm{i})$ and $H(h)(i)$ to obtain inequality (44). This means that $(u, \lambda) \in K \times Y$ also solves problem (41) and (5).

(ii) Assume that $(u, \lambda) \in K \times Y$ is a solution to problem (4) and (5). The inequality (8) can be obtained readily by multiplying inequality (5) by -1 and then summing it up with (4).

Conversely, if $(u, \lambda) \in K \times Y$ is a solution to problem (8), then inequalities (4) and (5) are a direct consequence of (8) via inserting $\rho=\lambda$ and $v=u$ into (8), respectively.

(iii) From assertion (i), it remains to show that $(u, \lambda) \in K \times Y$ is a solution to problem (6) and (7) if and only if it solves problem (9). Indeed, if $(u, \lambda) \in K \times Y$ is a solution to (6) and (17), then the inequality (9) is obtained easily via multiplying inequality (7) by -1 and by summing up the resulting inequality with (6).

For the converse, we put $\rho=\lambda$ and $v=u$ in (9), respectively, to obtain inequalities (6) and (77).

The following theorem delivers a crucial existence result for Problem 1 without any compactness hypothesis on operator $\gamma$.

TheOrem 10. Let $\Lambda$ be a nonempty, closed and convex subset of $E$ with $0_{E} \in \Lambda$. If hypotheses $H(A), H(b), H(J), H(h)(i)$-(ii), and $H(\gamma)$ are satisfied, then Problem 1 has at least one solution $(u, \lambda) \in V \times \Lambda$.

Proof. It follows from Theorem 9 that it is enough to prove that problem (8) admits a solution with $K=V$ and $Y=\Lambda$. The proof will be carried out in three steps.

Let $r, s \in \mathbb{N}$. We define sets $K(r)$ and $Y(s)$ by

$$
K(r):=\bar{B}_{V}(r) \subset V \quad \text { and } \quad Y(s):=\bar{B}_{E}(s) \cap \Lambda \subset \Lambda,
$$

where $\bar{B}_{H}(r)$ stands for a closed ball with centre $0_{H}$ and radius $r>0$ in a space $H$.

Step 1. For all $r, s \in \mathbb{N}$ fixed, the following problem admits a solution $\left(u_{r}, \lambda_{s}\right) \in$ $K(r) \times Y(s)$ such that

$$
\left\{\begin{array}{r}
\left\langle A u_{r}, v-u_{r}\right\rangle+b\left(v, \lambda_{s}\right)-b\left(u_{r}, \rho\right)+J^{0}\left(\gamma u_{r} ; \gamma v-\gamma u_{r}\right) \\
\geq\left\langle f, v-u_{r}\right\rangle \text { for all } v \in K(r) \text { and all } \rho \in Y(s) .
\end{array}\right.
$$

We consider a multivalued mapping $G: K(r) \times Y(s) \rightarrow 2^{K(r) \times Y(s)}$ given by

$$
\begin{aligned}
G(v, \rho):=\{( & (u, \lambda) \in K(r) \times Y(s) \mid\langle A v, v-u\rangle+b(v, \lambda)-b(u, \rho) \\
& \left.+\inf _{\xi_{v} \in \partial J(\gamma v)}\left\langle\xi_{v}, \gamma(v-u)\right\rangle_{X^{*} \times X} \geq\langle f, v-u\rangle+h(v-u)\right\} .
\end{aligned}
$$

It is obvious that for each $(v, \rho) \in K(r) \times Y(s)$, the set $G(v, \rho)$ is nonempty, since $(v, \rho) \in G(v, \rho)$. We now demonstrate that for each $(v, \rho) \in K(r) \times Y(s)$ fixed, the set $G(v, \rho)$ is weakly closed. Let $\left\{\left(u_{n}, \lambda_{n}\right)\right\} \subset G(v, \rho)$ be a sequence such that $u_{n} \rightarrow u$ weakly in $V$ and $\lambda_{n} \rightarrow \lambda$ weakly in $E$. We have

$$
\begin{aligned}
& \left\langle A v, v-u_{n}\right\rangle+b\left(v, \lambda_{n}\right)-b\left(u_{n}, \rho\right)+\left\langle\xi_{v}, \gamma\left(v-u_{n}\right)\right\rangle_{X^{*} \times X} \\
& \quad \geq\left\langle f, v-u_{n}\right\rangle+h\left(v-u_{n}\right)
\end{aligned}
$$


for all $\xi_{v} \in \partial J(\gamma v)$. Passing to the upper limit, as $n \rightarrow \infty$, in the above inequality, from $H(h)($ ii), one has

$$
\begin{aligned}
\langle f, & v-u\rangle+h(v-u) \\
& \leq \lim _{n \rightarrow \infty}\left\langle f, v-u_{n}\right\rangle+\limsup _{n \rightarrow \infty} h\left(v-u_{n}\right) \\
& =\limsup _{n \rightarrow \infty}\left[\left\langle f, v-u_{n}\right\rangle+h\left(v-u_{n}\right)\right] \\
& \leq \limsup _{n \rightarrow \infty}\left[\left\langle A v, v-u_{n}\right\rangle+b\left(v, \lambda_{n}\right)-b\left(u_{n}, \rho\right)+\left\langle\xi_{v}, \gamma\left(v-u_{n}\right)\right\rangle_{X^{*} \times X}\right] \\
& =\langle A v, v-u\rangle+b(v, \lambda)-b(u, \rho)+\left\langle\xi_{v}, \gamma(v-u)\right\rangle_{X^{*} \times X}
\end{aligned}
$$

for all $\xi_{v} \in \partial J(\gamma v)$. Hence,

$$
\begin{aligned}
& \langle f, v-u\rangle+h(v-u) \\
& \quad \leq\langle A v, v-u\rangle+b(v, \lambda)-b(u, \rho)+\inf _{\xi_{v} \in \partial J(\gamma v)}\left\langle\xi_{v}, \gamma(v-u)\right\rangle_{X^{*} \times X} .
\end{aligned}
$$

This means that $(u, \lambda) \in G(v, \rho)$, therefore, the set $G(v, \rho)$ is weakly closed. Note that $K(r) \times Y(s)$ is a nonempty, bounded, closed, and convex subset of $V \times E$. Moreover, from the reflexivity of $V \times E$, it follows that the set $K(r) \times Y(s)$ is weakly compact and convex. This ensures that $G(v, \rho)$ is relatively weakly compact in $V \times E$ for all $(v, \rho) \in V \times E$. Thus, we conclude that for all $(v, \rho) \in V \times E$, the set $G(v, \rho)$ is weakly compact, owning to the weak closedness of $G(v, \rho)$.

Now, we can distinguish two cases: (a) $G$ is a KKM mapping, and (b) $G$ is not a KKM mapping.

If case (a) occurs, then via invoking the F-KKM principle, Theorem 7 , we are able to find $\left(u_{r}, \lambda_{s}\right) \in K(r) \times Y(s)$ such that

$$
\left(u_{r}, \lambda_{s}\right) \in \bigcap_{(v, \rho) \in K(r) \times Y(s)} G(v, \rho) \neq \emptyset,
$$

that is,

$$
\left\langle A v, v-u_{r}\right\rangle+b\left(v, \lambda_{s}\right)-b\left(u_{r}, \rho\right)+\left\langle\xi_{v}, \gamma\left(v-u_{r}\right)\right\rangle_{X^{*} \times X} \geq\left\langle f, v-u_{r}\right\rangle+h\left(v-u_{r}\right)
$$

for all $\xi_{v} \in \partial J(\gamma v)$ and $(v, \rho) \in K(r) \times Y(s)$. Hence, we have

$$
\left\langle A v, v-u_{r}\right\rangle+b\left(v, \lambda_{s}\right)-b\left(u_{r}, \rho\right)+J^{0}\left(\gamma v ; \gamma v-\gamma u_{r}\right) \geq\left\langle f, v-u_{r}\right\rangle+h\left(v-u_{r}\right)
$$

for all $(v, \rho) \in K(r) \times Y(s)$. We now employ Theorem 9 to show that $\left(u_{r}, \lambda_{s}\right) \in$ $K(r) \times Y(s)$ solves problem (10).

On the other hand, when (b) holds, then there exist

$$
\left\{\left(v_{1}, \rho_{1}\right),\left(v_{2}, \rho_{2}\right), \ldots,\left(v_{N}, \rho_{N}\right)\right\} \subset K(r) \times Y(s)
$$


and $\left(u_{r}, \lambda_{s}\right) \in K(r) \times Y(s)$ with $u_{r}=\sum_{i=1}^{N} t_{i} v_{i}, \lambda_{s}=\sum_{i=1}^{N} t_{i} \rho_{i}, t_{i} \in[0,1]$ for $i=1,2, \ldots, N$, and $\sum_{i=1}^{N} t_{i}=1$ such that

$$
\left(u_{r}, \lambda_{s}\right) \notin \bigcup_{i=1}^{N} G\left(v_{i}, \rho_{i}\right) .
$$

This means that

$$
\begin{aligned}
& \left\langle A v_{i}, v_{i}-u_{r}\right\rangle+b\left(v_{i}, \lambda_{s}\right)-b\left(u_{r}, \rho_{i}\right)+\inf _{\xi_{i} \in \partial J\left(\gamma v_{i}\right)}\left\langle\xi_{i}, \gamma\left(v_{i}-u_{r}\right)\right\rangle_{X^{*} \times X} \\
& \quad<\left\langle f, v_{i}-u_{r}\right\rangle+h\left(v_{i}-u_{r}\right)
\end{aligned}
$$

for $i=1,2, \ldots, N$.

Claim 1. There exists a neighborhood $O$ of $\left(u_{r}, \lambda_{s}\right)$ in $V \times \Lambda$ such that whenever $(v, \rho) \in O \cap(K(r) \times Y(s))$, there holds

$$
\begin{aligned}
& \left\langle A v_{i}, v_{i}-v\right\rangle+b\left(v_{i}, \rho\right)-b\left(v, \rho_{i}\right)+\inf _{\xi_{i} \in \partial J\left(\gamma v_{i}\right)}\left\langle\xi_{i}, \gamma\left(v_{i}-v\right)\right\rangle_{X^{*} \times X} \\
& \quad<\left\langle f, v_{i}-v\right\rangle+h\left(v_{i}-v\right) .
\end{aligned}
$$

Arguing by contradiction, we may assume that there are $\left\{u_{n}\right\} \subset K(r),\left\{\lambda_{n}\right\} \subset$ $Y(s)$, and $\left\{j_{n}\right\} \subset\{1,2, \ldots, N\}$ such that $u_{n} \rightarrow u_{r}$ in $V, \lambda_{n} \rightarrow \lambda_{s}$ in $E$ and, for every $n \in \mathbb{N}$, we have

$$
\begin{aligned}
& \left\langle A v_{j_{n}}, v_{j_{n}}-u_{n}\right\rangle+b\left(v_{j_{n}}, \lambda_{n}\right)-b\left(u_{n}, \rho_{j_{n}}\right)+\left\langle\xi_{j_{n}}, \gamma\left(v_{j_{n}}-u_{n}\right)\right\rangle_{X^{*} \times X} \\
& \quad \geq\left\langle f, v_{j_{n}}-u_{n}\right\rangle+h\left(v_{j_{n}}-u_{n}\right)
\end{aligned}
$$

for all $\xi_{j_{n}} \in \partial J\left(\gamma v_{j_{n}}\right)$. Since for all $n \in \mathbb{N}, j_{n} \in\{1,2, \ldots, N\}$, so, without any loss of generality, we may suppose that there exists $j_{0} \in\{1,2, \ldots, N\}$ such that for all $n \in \mathbb{N}$, the following inequality holds

$$
\begin{aligned}
& \left\langle A v_{j_{0}}, v_{j_{0}}-u_{n}\right\rangle+b\left(v_{j_{0}}, \lambda_{n}\right)-b\left(u_{n}, \rho_{j_{0}}\right)+\left\langle\xi_{j_{0}}, \gamma\left(v_{j_{0}}-u_{n}\right)\right\rangle_{X^{*} \times X} \\
& \quad \geq\left\langle f, v_{j_{0}}-u_{n}\right\rangle+h\left(v_{j_{0}}-u_{n}\right)
\end{aligned}
$$

for all $\xi_{j_{0}} \in \partial J\left(\gamma v_{j_{0}}\right)$. If, we now pass to the upper limit, as $n \rightarrow \infty$, in the above inequality, we get

$$
\begin{aligned}
& \left\langle f, v_{j_{0}}-u_{r}\right\rangle+h\left(v_{j_{0}}-u_{r}\right) \\
& \quad \leq \lim _{n \rightarrow \infty}\left\langle f, v_{j_{0}}-u_{n}\right\rangle+\limsup _{n \rightarrow \infty} h\left(v_{j_{0}}-u_{n}\right) \\
& \quad=\limsup _{n \rightarrow \infty}\left[\left\langle f, v_{j_{0}}-u_{n}\right\rangle+h\left(v_{j_{0}}-u_{n}\right)\right] \\
& \quad \leq \limsup _{n \rightarrow \infty}\left[\left\langle A v_{j_{0}}, v_{j_{0}}-u_{n}\right\rangle+b\left(v_{j_{0}}, \lambda_{n}\right)-b\left(u_{n}, \rho_{j_{0}}\right)+\left\langle\xi_{j_{0}}, \gamma\left(v_{j_{0}}-u_{n}\right)\right\rangle_{X^{*} \times X}\right] \\
& \quad=\left\langle A v_{j_{0}}, v_{j_{0}}-u_{r}\right\rangle+b\left(v_{j_{0}}, \lambda_{s}\right)-b\left(u_{r}, \rho_{j_{0}}\right)+\left\langle\xi_{j_{0}}, \gamma\left(v_{j_{0}}-u_{r}\right)\right\rangle_{X^{*} \times X}
\end{aligned}
$$


for all $\xi_{j_{0}} \in \partial J\left(\gamma v_{j_{0}}\right)$, that is,

$$
\begin{aligned}
& \left\langle A v_{j_{0}}, v_{j_{0}}-u_{r}\right\rangle+b\left(v_{j_{0}}, \lambda_{s}\right)-b\left(u_{r}, \rho_{j_{0}}\right)+\inf _{\xi_{j_{0}} \in \partial J\left(\gamma v_{j_{0}}\right)}\left\langle\xi_{j_{0}}, \gamma\left(v_{j_{0}}-u_{r}\right)\right\rangle_{X^{*} \times X} \\
& \quad \geq\left\langle f, v_{j_{0}}-u_{r}\right\rangle+h\left(v_{j_{0}}-u_{r}\right) .
\end{aligned}
$$

This is a contradiction with (12), so, Claim 1 is valid.

Subsequently, from Claim 1 , for every $i \in\{1,2, \ldots, N\}$, we are able to find $\bar{\xi}_{i} \in$ $\partial J\left(\gamma v_{i}\right)$ such that

$$
\begin{aligned}
& \left\langle A v_{i}, v_{i}-v\right\rangle+b\left(v_{i}, \rho\right)-b\left(v, \rho_{i}\right)+\left\langle\bar{\xi}_{i}, \gamma\left(v_{i}-v\right)\right\rangle_{X^{*} \times X} \\
& \quad \leq\left\langle f, v_{i}-v\right\rangle+h\left(v_{i}-v\right)
\end{aligned}
$$

for all $(v, \rho) \in O \cap(K(r) \times Y(s))$. It follows from the $h$-relaxed monotonicity of operator $A \cdot+\gamma^{*} \partial J(\gamma \cdot)$ that

$$
\begin{aligned}
& \left\langle A v, v_{i}-v\right\rangle+b\left(v_{i}, \rho\right)-b\left(v, \rho_{i}\right)+\left\langle\xi_{v}, \gamma\left(v_{i}-v\right)\right\rangle_{X^{*} \times X}+h\left(v_{i}-v\right) \\
& \quad \leq\left\langle A v_{i}, v_{i}-v\right\rangle+b\left(v_{i}, \rho\right)-b\left(v, \rho_{i}\right)+\left\langle\bar{\xi}_{i}, \gamma\left(v_{i}-v\right)\right\rangle_{X^{*} \times X} \\
& \quad \leq\left\langle f, v_{i}-v\right\rangle+h\left(v_{i}-v\right)
\end{aligned}
$$

for all $\xi_{v} \in \partial J(\gamma v)$ and $(v, \rho) \in O \cap(K(r) \times Y(s))$, therefore,

$$
\left\langle A v, v-v_{i}\right\rangle+b\left(v, \rho_{i}\right)-b\left(v_{i}, \rho\right)+\left\langle\xi_{v}, \gamma\left(v-v_{i}\right)\right\rangle_{X^{*} \times X} \geq\left\langle f, v-v_{i}\right\rangle
$$

for all $\xi_{v} \in \partial J(\gamma v)$ and $(v, \rho) \in O \cap(K(r) \times Y(s))$. Next, multiplying the above inequality by $t_{i} \geq 0$, and summing up those inequalities from $i=1$ to $N$, one obtains

$$
\left\langle A v, v-u_{r}\right\rangle+b\left(v, \lambda_{s}\right)-b\left(u_{r}, \rho\right)+\left\langle\xi_{v}, \gamma\left(v-u_{r}\right)\right\rangle_{X^{*} \times X} \geq\left\langle f, v-u_{r}\right\rangle
$$

for all $\xi_{v} \in \partial J(\gamma v)$ and $(v, \rho) \in O \cap(K(r) \times Y(s))$, where we have used the facts $u_{r}=\sum_{i=1}^{N} t_{i} v_{i}, \lambda_{s}=\sum_{i=1}^{N} t_{i} \rho_{i}, t_{i} \in[0,1]$ for $i=1,2, \ldots, N$, and $\sum_{i=1}^{N} t_{i}=1$. Assume now that $(w, \eta) \in K(r) \times Y(s)$ is arbitrary, and consider the sequence $\left\{\left(v_{n}, \rho_{n}\right)\right\} \subset$ $V \times E$ defined by

$$
v_{n}:=\frac{1}{n} w+\left(1-\frac{1}{n}\right) u_{r} \quad \text { and } \quad \rho_{n}:=\frac{1}{n} \eta+\left(1-\frac{1}{n}\right) \lambda_{s} .
$$

It is not difficult to find $N_{1} \in \mathbb{N}$ large enough such that $\left(v_{n}, \rho_{n}\right) \in O \cap(K(r) \times Y(s))$ for all $n \geq N_{1}$. Inserting $v=v_{n}$ and $\rho=\rho_{n}$ into (14), it reads

$$
\begin{aligned}
\frac{1}{n}\left\langle f, w-u_{r}\right\rangle & \\
\leq & \frac{1}{n}\left\langle A v_{n}, w-u_{r}\right\rangle+\frac{1}{n} b\left(w, \lambda_{s}\right)+\left(1-\frac{1}{n}\right) b\left(u_{r}, \lambda_{s}\right)-\frac{1}{n} b\left(u_{r}, \eta\right) \\
& -\left(1-\frac{1}{n}\right) b\left(u_{r}, \lambda_{s}\right)+\frac{1}{n}\left\langle\xi_{v_{n}}, \gamma\left(w-u_{r}\right)\right\rangle_{X^{*} \times X} \quad\left(\text { for all } \xi_{v_{n}} \in \partial J\left(\gamma v_{n}\right)\right) \\
\leq & \frac{1}{n}\left[\left\langle A v_{n}, w-u_{r}\right\rangle+b\left(w, \lambda_{s}\right)-b\left(u_{r}, \eta\right)+J^{0}\left(\gamma v_{n} ; \gamma\left(w-u_{r}\right)\right)\right]
\end{aligned}
$$


for all $n \geq N_{1}$. If we divide both sides of the above inequality by $\frac{1}{n}$, and then pass to the upper limit, as $n \rightarrow \infty$, we get

$$
\begin{aligned}
& \left\langle f, w-u_{r}\right\rangle \\
& \quad \leq \limsup _{n \rightarrow \infty}\left[\left\langle A v_{n}, w-u_{r}\right\rangle+b\left(w, \lambda_{s}\right)-b\left(u_{r}, \eta\right)+J^{0}\left(\gamma v_{n} ; \gamma\left(w-u_{r}\right)\right)\right] \\
& \quad \leq \limsup _{n \rightarrow \infty}\left\langle A v_{n}, w-u_{r}\right\rangle+b\left(w, \lambda_{s}\right)-b\left(u_{r}, \eta\right)+\limsup _{n \rightarrow \infty} J^{0}\left(\gamma v_{n} ; \gamma\left(w-u_{r}\right)\right) \\
& \quad \leq\left\langle A u_{r}, w-u_{r}\right\rangle+b\left(w, \lambda_{s}\right)-b\left(u_{r}, \eta\right)+J^{0}\left(\gamma u_{r} ; \gamma\left(w-u_{r}\right)\right) .
\end{aligned}
$$

Since $w \in K(r)$ and $\eta \in Y(s)$ are arbitrary, we conclude that $\left(u_{r}, \lambda_{s}\right) \in K(r) \times Y(s)$ is also a solution to problem (10).

Step 2. For every $s \in \mathbb{N}$ fixed, the following problem has at least one solution $\left(u_{s}, \lambda_{s}\right) \in V \times Y(s)$ such that

$$
\left\{\begin{array}{c}
\left\langle A u_{s}, v-u_{s}\right\rangle+b\left(v, \lambda_{s}\right)-b\left(u_{s}, \rho\right)+J^{0}\left(\gamma u_{s} ; \gamma v-\gamma u_{s}\right) \\
\geq\left\langle f, v-u_{s}\right\rangle \text { for all } v \in V \text { and all } \rho \in Y(s) .
\end{array}\right.
$$

It follows from Step 1 that for any $r, s \in \mathbb{N}$, problem (10) admits a solution $\left(u_{r}, \lambda_{s}\right) \in K(r) \times Y(s)$.

Claim 2. There exist $r_{0} \in \mathbb{N}$ and a solution $\left(u_{r_{0}}, \lambda_{s}\right) \in K\left(r_{0}\right) \times Y(s)$ to problem (10) for $r=r_{0}$ such that

$$
\left\|u_{r_{0}}\right\|_{V}<r_{0}
$$

Suppose that this claim is not true, so for any $r \in \mathbb{N}$, for each solution $\left(u_{r}, \lambda_{s}\right) \in$ $K(r) \times Y(s)$ of problem (10), it holds

$$
\left\|u_{r}\right\|_{V}=r
$$

Since $0_{E} \in Y(s)$ and $0_{V} \in K(r)$ for all $r \in \mathbb{N}$, we now take $v=0_{V}$ and $\rho=0_{E}$ in (10) to obtain

$$
\begin{aligned}
& \left\langle A u_{r}, u_{r}\right\rangle \leq b\left(0_{V}, \lambda\right)-b\left(u_{r}, 0_{E}\right)+J^{0}\left(\gamma u_{r} ;-\gamma u_{r}\right)+\left\langle f, u_{r}\right\rangle \\
& \quad=J^{0}\left(\gamma u_{r} ;-\gamma u_{r}\right)+\left\langle f, u_{r}\right\rangle \leq \alpha_{J}+\beta_{J}\|\gamma\|_{\mathcal{L}(V, X)}^{\theta}\left\|u_{r}\right\|_{V}^{\theta}+\|f\|_{V^{*}}\left\|u_{r}\right\|_{V}
\end{aligned}
$$

and hence,

$$
\frac{\left\langle A u_{r}, u_{r}\right\rangle}{\left\|u_{r}\right\|_{V}^{\max \{\theta, 1\}}} \leq \frac{\alpha_{J}}{\left\|u_{r}\right\|_{V}^{\max \{\theta, 1\}}}+\frac{\beta_{J}\left\|_{\gamma}\right\|_{\mathcal{L}(V, X)}^{\theta}}{\left\|u_{r}\right\|_{V}^{\max \{0,1-\theta\}}}+\frac{\|f\|_{V^{*}}}{\left\|u_{r}\right\|_{V}^{\max \{0, \theta-1\}}}
$$

Passing to the limit, as $r \rightarrow \infty$, and invoking the coercivity condition $H(A)$ (iii), we get a contradiction. This ensures that Claim 2 is true.

Assume now that $\left(u_{r_{0}}, \lambda_{s}\right) \in K\left(r_{0}\right) \times Y(s)$ is a solution to problem (10) for $r=r_{0}$ such that inequality (16) holds. We affirm that $\left(u_{r_{0}}, \lambda_{s}\right) \in V \times Y(s)$ is also a solution 
to problem (15). Let $w \in V$ and $\eta \in Y(s)$ be arbitrary, and $t \in(0,1)$ be small enough such that $v_{t}=t w+(1-t) u_{r_{0}} \in K\left(r_{0}\right)$ (thanks to inequality (16)). Putting $v=v_{t}$ and $\rho=\rho_{t}:=t \eta+(1-t) \lambda_{s}$ into (10), it holds

$$
\begin{aligned}
& t\left\langle f, w-u_{r_{0}}\right\rangle \\
& \leq t\left\langle A u_{r_{0}}, w-u_{r_{0}}\right\rangle+t b\left(w, \lambda_{s}\right)+(1-t) b\left(u_{r_{0}}, \lambda_{s}\right)-t b\left(u_{r_{0}}, \eta\right) \\
& \quad-(1-t) b\left(u_{r_{0}}, \lambda_{s}\right)+t J^{0}\left(\gamma u_{r_{0}} ; \gamma w-\gamma u_{r_{0}}\right) \\
& =t\left[\left\langle A u_{r_{0}}, w-u_{r_{0}}\right\rangle+b\left(w, \lambda_{s}\right)-b\left(u_{r_{0}}, \eta\right)+J^{0}\left(\gamma u_{r_{0}} ; \gamma w-\gamma u_{r_{0}}\right)\right],
\end{aligned}
$$

and

$$
\left\langle A u_{r_{0}}, w-u_{r_{0}}\right\rangle+b\left(w, \lambda_{s}\right)-b\left(u_{r_{0}}, \eta\right)+J^{0}\left(\gamma u_{r_{0}} ; \gamma w-\gamma u_{r_{0}}\right) \geq\left\langle f, w-u_{r_{0}}\right\rangle
$$

for all $w \in V$ and $\eta \in Y(s)$. Consequently, $\left(u_{r_{0}}, \lambda_{s}\right) \in V \times Y(s)$ is a solution to problem (15).

Step 3. Problem 1 has at least one solution.

Indeed, Step 2 guarantees that for each $s \in \mathbb{N}$, problem (15) admits a solution $\left(u_{s}, \lambda_{s}\right) \in V \times Y(s)$.

Claim 3. The sequences $\left\{u_{s}\right\}$ and $\left\{\lambda_{s}\right\}$ are both uniformly bounded in $V$ and $E$, respectively.

As concerns the sequence $\left\{u_{s}\right\}$, if it is unbounded, then, without any loss of generality, we may assume that $\left\|u_{s}\right\|_{V} \rightarrow+\infty$, as $s \rightarrow \infty$. Inserting $v=0_{V}$ and $\rho=0_{E}$ into (15), it has

$$
\begin{gathered}
\left\langle A u_{s}, u_{s}\right\rangle \leq b\left(0_{V}, \lambda_{s}\right)-b\left(u_{s}, 0_{E}\right)+J^{0}\left(\gamma u_{s} ;-\gamma u_{s}\right)+\left\langle f, u_{s}\right\rangle \\
\leq \alpha_{J}+\beta_{J}\|\gamma\|_{\mathcal{L}(V, X)}^{\theta}\left\|u_{s}\right\|_{V}^{\theta}+\|f\|_{V^{*}}\left\|u_{s}\right\|_{V},
\end{gathered}
$$

and

$$
\frac{\left\langle A u_{s}, u_{s}\right\rangle}{\left\|u_{s}\right\|_{V}^{\max \{\theta, 1\}}} \leq \frac{\alpha_{J}}{\left\|u_{s}\right\|_{V}^{\max \{\theta, 1\}}}+\frac{\beta_{J}\left\|^{\prime}\right\|_{\mathcal{L}(V, X)}^{\theta}}{\left\|u_{s}\right\|_{V}^{\max \{0,1-\theta\}}}+\frac{\|f\|_{V^{*}}}{\left\|u_{s}\right\|_{V}^{\max \{0, \theta-1\}}}
$$

Now, the coercivity condition $H(A)$ (iii) concludes a contradiction, so the sequence $\left\{u_{s}\right\}$ is uniformly bounded in $V$.

It remains to show that the sequence $\left\{\lambda_{s}\right\}$ is uniformly bounded in $E$ too. For any $w \in V \backslash\left\{0_{V}\right\}$, inserting $v=u_{s}-\frac{w}{\|w\|_{V}}$ and $\rho=\lambda_{s}$ into (15), we have

$$
\begin{aligned}
& b\left(\frac{w}{\|w\|_{V}}, \lambda_{s}\right) \leq\left\langle A u_{s},-\frac{w}{\|w\|_{V}}\right\rangle+J^{0}\left(\gamma u_{s} ;-\gamma \frac{w}{\|w\|_{V}}\right)+\left\langle f, \frac{w}{\|w\|_{V}}\right\rangle \\
& =\left\langle A u_{s},-\frac{w}{\|w\|_{V}}\right\rangle+\max _{\xi u_{s} \in \partial J\left(\gamma u_{s}\right)}\left\langle\xi_{u_{s}},-\gamma \frac{w}{\|w\|_{V}}\right\rangle_{X^{*} \times X}+\left\langle f, \frac{w}{\|w\|_{V}}\right\rangle \\
& \quad \leq\left\|A u_{s}\right\|_{V^{*}}+\left\|\gamma^{*} \partial J\left(\gamma u_{s}\right)\right\|_{V^{*}}+\|f\|_{V^{*}} .
\end{aligned}
$$


Passing to supremum with $w \in V \backslash\left\{0_{V}\right\}$, by using inequality (3), we deduce

$$
\alpha_{b}\left\|\lambda_{s}\right\|_{E} \leq \limsup _{w \in V \backslash\left\{0_{V}\right\}} b\left(\frac{w}{\|w\|_{V}}, \lambda_{s}\right) \leq\left\|A u_{s}\right\|_{V^{*}}+\left\|\gamma^{*} \partial J\left(\gamma u_{s}\right)\right\|_{V^{*}}+\|f\|_{V^{*}}
$$

for all $s \in \mathbb{N}$. This inequality combined with the uniform boundedness of $\left\{u_{s}\right\}$, hypotheses $H(A)$ (iv) and $H(J)$ (iii), implies that the sequence $\left\{\lambda_{s}\right\}$ is uniformly bounded in $E$.

From Claim 3, we can find $s_{0} \in \mathbb{N}$ large enough such that $\left\|\lambda_{s_{0}}\right\|_{E}<s_{0}$. We shall verify that $\left(u_{s_{0}}, \lambda_{s_{0}}\right)$ is also a solution to Problem 1. Let $\eta \in \Lambda$ be arbitrary and $t \in(0,1)$ be small enough such that $\rho_{t}=t \eta+(1-t) \lambda_{s_{0}} \in Y\left(s_{0}\right)$. After inserting $\rho=\rho_{t}$ and $v=u_{s_{0}}$ into (15) for $s=s_{0}$, we have

$$
b\left(u_{s_{0}}, \lambda_{s_{0}}\right)-t b\left(u_{s_{0}}, \eta\right)-(1-t) b\left(u_{s_{0}}, \lambda_{s_{0}}\right) \geq 0
$$

i.e.,

$$
b\left(u_{s_{0}}, \eta-\lambda_{s_{0}}\right) \leq 0 \text { for all } \eta \in \Lambda .
$$

Putting $\rho=\lambda_{s_{0}}$ into (15), we have

$$
\left\langle A u_{s_{0}}, v-u_{s_{0}}\right\rangle+b\left(v-u_{s_{0}}, \lambda_{s_{0}}\right)+J^{0}\left(\gamma u_{s_{0}} ; \gamma v-\gamma u_{s_{0}}\right) \geq\left\langle f, v-u_{s_{0}}\right\rangle \text { for all } v \in V \text {. }
$$

The last two inequalities reveal that $\left(u_{s_{0}}, r_{s_{0}}\right) \in V \times \Lambda$ is a solution to Problem 1, which concludes the proof.

In what follows, we denote the solution set to Problem 1 by $S(A, J, b, f)$. By Theorem 10, we know that the set $S(A, J, b, f) \subset V \times \Lambda$ is nonempty. It is desirable to investigate further properties of the solution set which can be useful, for instance, in the study of optimal control problems for systems governed by a mixed variationalhemivariational inequality. This is the reason that in the next theorem we study essential properties of $S(A, J, b, f)$, such as, convexity, closedness and continuity.

THEOREM 11. Let $\Lambda$ be a nonempty, closed and convex subset of $E$ with $0_{E} \in \Lambda$. If hypotheses $H(A), H(b), H(J), H(h)(i)-(i i)$, and $H(\gamma)$ are satisfied, then the following hold

(i) the solution set $S(A, J, b, f)$ is bounded and weakly closed in $V \times \Lambda$,

(ii) if $h: V \rightarrow \mathbb{R}$ is convex, then the set $S(A, J, b, f)$ is convex as well,

(iii) the multivalued mapping $\mathcal{S}: V^{*} \rightarrow 2^{V \times \Lambda}$, defined by $f \mapsto \mathcal{S}(f):=S(A, J, b, f)$, is bounded, i.e., $\mathcal{S}$ maps bounded subsets of $V^{*}$ to bounded subsets of $V \times \Lambda$,

(iv) the multivalued mapping $f \mapsto \mathcal{S}(f)$ is strongly-weakly upper semicontinuous, (i.e., it is upper semicontinuous from $V^{*}$ endowed with the norm topology to the subsets of $V \times \Lambda$ endowed with the weak topology), and it has a strongly-weakly closed graph.

Proof. (i) Arguing by contradiction, if we assume that $S(A, J, b, f)$ is unbounded, then there exists a sequence $\left\{\left(u_{n}, \lambda_{n}\right)\right\} \subset S(A, J, b, f)$ such that

$$
\left\|u_{n}\right\|_{V}+\left\|\lambda_{n}\right\|_{E} \rightarrow+\infty, \text { as } n \rightarrow \infty
$$


For the sequence $\left\{\left(u_{n}, \lambda_{n}\right)\right\}$, we claim that $\left\{u_{n}\right\}$ is bounded in $V$. If it is not true, then one has

$$
\left\|u_{n}\right\|_{V} \rightarrow+\infty, \text { as } n \rightarrow \infty .
$$

In fact, for each $n \in \mathbb{N}$, we have

$$
\begin{aligned}
& \left\langle A\left(u_{n}\right), v-u_{n}\right\rangle+b\left(v-u_{n}, \lambda_{n}\right)+J^{0}\left(\gamma u_{n} ; \gamma v-\gamma u_{n}\right) \geq\left\langle f, v-u_{n}\right\rangle \text { for all } v \in V, \\
& b\left(u_{n}, \rho-\lambda_{n}\right) \leq 0 \text { for all } \rho \in \Lambda .
\end{aligned}
$$

Choosing $v=0_{V}$ and $\rho=0_{E}$ in (19) and (20), respectively, from the resulting inequalities, it yields

$$
\begin{aligned}
& \left\langle A\left(u_{n}\right), u_{n}\right\rangle \leq b\left(-u_{n}, \lambda_{n}\right)+J^{0}\left(\gamma u_{n} ;-\gamma u_{n}\right)+\left\langle f, u_{n}\right\rangle \\
& \leq \alpha_{J}+\beta_{J}\|\gamma\|_{\mathcal{L}(V, X)}^{\theta}\left\|u_{n}\right\|_{V}^{\theta}+\|f\|_{V^{*}}\left\|u_{n}\right\|_{V},
\end{aligned}
$$

and hence

$$
\frac{\left\langle A\left(u_{n}\right), u_{n}\right\rangle}{\left\|u_{n}\right\|_{V}^{\max \{1, \theta\}}} \leq \frac{\alpha_{J}}{\left\|u_{n}\right\|_{V}^{\max \{1, \theta\}}}+\frac{\beta_{J}\|\gamma\|_{\mathcal{L}(V, X)}^{\theta}}{\left\|u_{n}\right\|_{V}^{\max \{1-\theta, 0\}}}+\frac{\|f\|_{V^{*}}}{\left\|u_{n}\right\|_{V}^{\max \{0, \theta-1\}}} .
$$

The above inequality combined with (18) and hypothesis $H(A)$ (iii) leads to a contradiction. Hence, we deduce that the sequence $\left\{u_{n}\right\}$ is bounded in $V$.

Moreover, by (17), we know that $\left\{\lambda_{n}\right\}$ is unbounded in $E$. Let $w \in V \backslash\left\{0_{V}\right\}$ be arbitrary. Taking account of $v=u_{n}-\frac{w}{\|w\|_{V}}$ in (19), it reads

$$
\begin{aligned}
& b\left(\frac{w}{\|w\|_{V}}, \lambda_{n}\right) \leq\left\langle A u_{n},-\frac{w}{\|w\|_{V}}\right\rangle+J^{0}\left(\gamma u_{n} ;-\gamma \frac{w}{\|w\|_{V}}\right)+\left\langle f, \frac{w}{\|w\|_{V}}\right\rangle \\
& =\left\langle A u_{n},-\frac{w}{\|w\|_{V}}\right\rangle+\max _{\xi_{n} \in \partial J\left(\gamma u_{n}\right)}\left\langle\xi_{u_{n}},-\gamma \frac{w}{\|w\|_{V}}\right\rangle_{X^{*} \times X}+\left\langle f, \frac{w}{\|w\|_{V}}\right\rangle \\
& \quad \leq\left\|A u_{n}\right\|_{V^{*}}+\left\|\gamma^{*} \partial J\left(\gamma u_{n}\right)\right\|_{V^{*}}+\|f\|_{V^{*}} .
\end{aligned}
$$

Passing to supremum with $w \in V \backslash\left\{0_{V}\right\}$ and using hypotheses $H(A)$ (iv), $H(J)($ iii), $H(b)$, and the boundedness of $\left\{u_{n}\right\}$, we are able to find a constant $C^{*}$, which is independent of $n$, such that

$$
\left\|\lambda_{n}\right\|_{E} \leq C^{*} \text { for all } n \in \mathbb{N} \text {. }
$$

This leads to a contradiction with (17). Therefore, we conclude that the solution set $S(A, J, b, f)$ to Problem 1 is bounded in $V \times \Lambda$.

Next, we show the weak closedness of $S(A, J, b, f)$. Let $\left\{\left(u_{n}, \lambda_{n}\right)\right\} \subset S(A, J, b, f)$ be a sequence such that

$$
u_{n} \rightarrow u \text { weakly in } V \text {, and } \lambda_{n} \rightarrow \lambda \text { weakly in } E \text {, as } n \rightarrow \infty \text {. }
$$

It follows from Theorem 9 that

$$
\begin{aligned}
& \left\langle A u_{n}, v-u_{n}\right\rangle+b\left(v, \lambda_{n}\right)-b\left(u_{n}, \rho\right)+J^{0}\left(\gamma u_{n} ; \gamma v-\gamma u_{n}\right) \\
& \geq\left\langle f, v-u_{n}\right\rangle \text { for all } v \in K \text { and all } \rho \in Y .
\end{aligned}
$$


Furthermore, the $h$-relaxed monotonicity of the mapping $u \mapsto A u+\gamma^{*} \partial J(\gamma u)$ implies

$$
\begin{aligned}
& \left\langle A u_{n}, v-u_{n}\right\rangle+J^{0}\left(\gamma u_{n} ; \gamma v-\gamma u_{n}\right)+h\left(v-u_{n}\right) \\
& \quad=\left\langle A u_{n}, v-u_{n}\right\rangle+\left\langle\xi_{u_{n}}, \gamma\left(v-u_{n}\right)\right\rangle_{X^{*} \times X}+h\left(v-u_{n}\right) \\
& \quad \leq\left\langle A v, v-u_{n}\right\rangle+\left\langle\xi_{v}, \gamma\left(v-u_{n}\right)\right\rangle_{X^{*} \times X}
\end{aligned}
$$

for all $\xi_{v} \in \partial J(\gamma v)$, where $\xi_{u_{n}} \in \partial J\left(\gamma u_{n}\right)$ is such that $\left\langle\xi_{u_{n}}, \gamma\left(v-u_{n}\right)\right\rangle_{X^{*} \times X}=$ $J^{0}\left(\gamma u_{n} ; \gamma v-\gamma u_{n}\right)$. We use the last two inequalities to obtain

$$
\begin{aligned}
& \left\langle A v, v-u_{n}\right\rangle+b\left(v, \lambda_{n}\right)-b\left(u_{n}, \rho\right)+\left\langle\xi_{v}, \gamma\left(v-u_{n}\right)\right\rangle_{X^{*} \times X} \\
& \geq\left\langle f, v-u_{n}\right\rangle+h\left(v-u_{n}\right) \text { for all } \xi_{v} \in \partial J(\gamma v),
\end{aligned}
$$

and all $(v, \rho) \in V \times \Lambda$. Passing to the upper limit, as $n \rightarrow \infty$, one has

$$
\begin{aligned}
\langle f, & v-u\rangle+h(v-u) \\
& \leq \limsup _{n \rightarrow \infty}\left[\left\langle f, v-u_{n}\right\rangle+h\left(v-u_{n}\right)\right] \\
& \leq \limsup _{n \rightarrow \infty}\left[\left\langle A v, v-u_{n}\right\rangle+b\left(v, \lambda_{n}\right)-b\left(u_{n}, \rho\right)+\left\langle\xi_{v}, \gamma\left(v-u_{n}\right)\right\rangle_{X^{*} \times X}\right] \\
& \leq\langle A v, v-u\rangle+b(v, \lambda)-b(u, \rho)+\left\langle\xi_{v}, \gamma(v-u)\right\rangle_{X^{*} \times X} \quad\left(\text { for all } \xi_{v} \in \partial J(\gamma v)\right) \\
& \leq\langle A v, v-u\rangle+b(v, \lambda)-b(u, \rho)+J^{0}(\gamma v ; \gamma v-\gamma u)
\end{aligned}
$$

for all $v \in V$ and $\rho \in \Lambda$. We now invoke Theorem 9 again to reveal that $(u, \lambda) \in$ $S(A, J, b, f)$. Hence, $S(A, J, b, f)$ is a weakly closed set.

(ii) Assume that $h$ is a convex function. Let $\left(u_{1}, \lambda_{1}\right),\left(u_{2}, \lambda_{2}\right) \in S(A, J, b, f)$ and $t \in(0,1)$. From Theorem 9, we have

$$
\begin{aligned}
& \left\langle A u_{i}, v-u_{i}\right\rangle+b\left(v, \lambda_{i}\right)-b\left(u_{i}, \rho\right)+J^{0}\left(\gamma u_{i} ; \gamma v-\gamma u_{i}\right) \\
& \quad \geq\left\langle f, v-u_{i}\right\rangle
\end{aligned}
$$

for all $v \in V$ and $\rho \in \Lambda$. Hence, for $i=1,2$, we get

$$
\begin{aligned}
& \left\langle A v, v-u_{i}\right\rangle+b\left(v, \lambda_{i}\right)-b\left(u_{i}, \rho\right)+\left\langle\xi_{v}, \gamma\left(v-u_{i}\right)\right\rangle_{X^{*} \times X} \\
& \quad \geq\left\langle f, v-u_{i}\right\rangle+h\left(v-u_{i}\right)
\end{aligned}
$$

for all $\xi_{v} \in \partial J(\gamma v)$ and $(v, \rho) \in V \times \Lambda$. Here, we have applied the $h$-relaxed monotonicity of $A \cdot+\gamma^{*} \partial J(\gamma \cdot)$. Denote $u_{t}=t u_{1}+(1-t) u_{2}$ and $\lambda_{t}=t \lambda_{1}+(1-t) \lambda_{2}$. It follows that

$$
\begin{aligned}
&\left\langle A v, v-u_{t}\right\rangle+b\left(v, \lambda_{t}\right)-b\left(u_{t}, \rho\right)+\left\langle\xi_{v}, \gamma\left(v-u_{t}\right)\right\rangle_{X^{*} \times X} \\
&= t\left[\left\langle A v, v-u_{1}\right\rangle+b\left(v, \lambda_{1}\right)-b\left(u_{1}, \rho\right)+\left\langle\xi_{v}, \gamma\left(v-u_{1}\right)\right\rangle_{X^{*} \times X}\right] \\
&+(1-t)\left[\left\langle A v, v-u_{2}\right\rangle+b\left(v, \lambda_{2}\right)-b\left(u_{2}, \rho\right)+\left\langle\xi_{v}, \gamma\left(v-u_{2}\right)\right\rangle_{X^{*} \times X}\right] \\
& \geq t\left\langle f, v-u_{1}\right\rangle+(1-t)\left\langle f, v-u_{2}\right\rangle+t h\left(v-u_{1}\right)+(1-t) h\left(v-u_{2}\right) \\
& \geq\left\langle f, v-u_{t}\right\rangle+h\left(v-u_{t}\right)
\end{aligned}
$$


for all $\xi_{v} \in \partial J(\gamma v)$ and $(v, \rho) \in V \times \Lambda$, where the last inequality is obtained by using the convexity of function $h$. Combining this inequality with Theorem 9 and the fact

$$
\begin{aligned}
& \left\langle A v, v-u_{t}\right\rangle+b\left(v, \lambda_{t}\right)-b\left(u_{t}, \rho\right)+J^{0}\left(\gamma v ; \gamma v-\gamma u_{t}\right) \\
& \quad \geq\left\langle A v, v-u_{t}\right\rangle+b\left(v, \lambda_{t}\right)-b\left(u_{t}, \rho\right)+\left\langle\xi_{v}, \gamma\left(v-u_{t}\right)\right\rangle_{X^{*} \times X} \quad\left(\text { for all } \xi_{v} \in \partial J(\gamma v)\right) \\
& \quad \geq\left\langle f, v-u_{t}\right\rangle+h\left(v-u_{t}\right) \quad \text { for all } v \in V \text { and } \rho \in \Lambda,
\end{aligned}
$$

implies that $\left(u_{t}, \lambda_{t}\right)$ is also a solution to Problem 1. This proves that $S(A, J, b, f)$ is a convex set.

(iii) If $\mathcal{S}: V^{*} \rightarrow 2^{V \times \Lambda}$ is not a bounded mapping, then we are able to find a bounded set $B \subset V^{*}$, and sequences $\left\{f_{n}\right\} \subset B,\left\{\left(u_{n}, \lambda_{n}\right)\right\} \subset V \times \Lambda$ with $\left(u_{n}, \lambda_{n}\right) \in \mathcal{S}\left(f_{n}\right)$ for all $n \in \mathbb{N}$ such that $\left\|u_{n}\right\|_{V}+\left\|\lambda_{n}\right\|_{E} \rightarrow+\infty$, as $n \rightarrow \infty$. As in the proof of assertion (i), we derive

$$
\frac{\left\langle A\left(u_{n}\right), u_{n}\right\rangle}{\left\|u_{n}\right\|_{V}^{\max \{1, \theta\}}} \leq \frac{\alpha_{J}}{\left\|u_{n}\right\|_{V}^{\max \{1, \theta\}}}+\frac{\beta\left\|_{J}\right\|^{\prime} \|_{\mathcal{L}(V, X)}^{\theta}}{\left\|u_{n}\right\|_{V}^{\max \{1-\theta, 0\}}}+\frac{\left\|f_{n}\right\|_{V^{*}}}{\left\|u_{n}\right\|_{V}^{\max \{0, \theta-1\}}},
$$

and

$$
b\left(\frac{w}{\|w\|_{V}}, \lambda_{n}\right) \leq\left\|A u_{n}\right\|_{V^{*}}+\left\|\gamma^{*} \partial J\left(\gamma u_{n}\right)\right\|_{V^{*}}+\left\|f_{n}\right\|_{V^{*}}
$$

The above inequalities combined with the boundedness of $\left\{f_{n}\right\}$ and the coercivity condition $H(A)$ (iii) leads to a contradiction. Consequently, $\mathcal{S}: V^{*} \rightarrow 2^{V \times \Lambda}$ is a bounded mapping.

(iv) In order to prove the upper semicontinuity of the mapping $f \mapsto \mathcal{S}(f)$, by Proposition 3, it is enough to prove that for each weakly closed subset $C$ of $V \times \Lambda$, the set

$$
\mathcal{S}^{-}(C):=\left\{f \in V^{*} \mid \mathcal{S}(f) \cap C \neq \emptyset\right\}
$$

is closed in $V^{*}$. Let $C$ be a weakly closed subset of $V \times \Lambda$, and $\left\{f_{n}\right\} \subset \mathcal{S}^{-}(C)$ be a sequence such that $f_{n} \rightarrow f$ in $V^{*}$. Thus, for each $n \in \mathbb{N}$, we can find a pair of elements $\left(u_{n}, \lambda_{n}\right) \in \mathcal{S}\left(f_{n}\right) \cap C$, i.e.,

$$
\begin{aligned}
& \left\langle A u_{n}, v-u_{n}\right\rangle+b\left(v, \lambda_{n}\right)-b\left(u_{n}, \rho\right)+J^{0}\left(\gamma u_{n} ; \gamma v-\gamma u_{n}\right) \\
& \geq\left\langle f_{n}, v-u_{n}\right\rangle \text { for all } v \in V \text { and all } \rho \in \Lambda .
\end{aligned}
$$

The $h$-relaxed monotonicity of $A \cdot+\gamma^{*} \partial J(\gamma \cdot)$ shows that

$$
\begin{aligned}
& \left\langle A v, v-u_{n}\right\rangle+b\left(v, \lambda_{n}\right)-b\left(u_{n}, \rho\right)+\left\langle\xi_{v}, \gamma\left(v-u_{n}\right)\right\rangle_{X^{*} \times X} \\
& \quad \geq\left\langle f_{n}, v-u_{n}\right\rangle+h\left(v-u_{n}\right)
\end{aligned}
$$

for all $\xi_{v} \in \partial J(\gamma v)$ and $(v, \rho) \in V \times \Lambda$. From assertion (iii), we can see that the sequence $\left\{\left(u_{n}, \lambda_{n}\right)\right\}$ is bounded in $V \times E$. The latter and the reflexivity of $V \times E$ guarantee that there exist a subsequence of $\left\{\left(u_{n}, \lambda_{n}\right)\right\}$, still denoted in the same way, and a pair of elements $(u, \lambda) \in V \times \Lambda$ such that

$$
u_{n} \rightarrow u \text { weakly in } V \text {, and } \lambda_{n} \rightarrow \lambda \text { weakly in } E \text {, as } n \rightarrow \infty \text {. }
$$


Taking into account the inequality (22), and passing to the upper limit, as $n \rightarrow \infty$, we obtain

$$
\begin{aligned}
& \langle A v, v-u\rangle+b(v, \lambda)-b(u, \rho)+J^{0}(\gamma v ; \gamma v-\gamma u) \\
& \quad \geq\langle A v, v-u\rangle+b(v, \lambda)-b(u, \rho)+\left\langle\xi_{v}, \gamma(v-u)\right\rangle_{X^{*} \times X} \quad\left(\text { for all } \xi_{v} \in \partial J(\gamma v)\right) \\
& \quad \geq\langle f, v-u\rangle+h(v-u)
\end{aligned}
$$

for all $(v, \rho) \in V \times \Lambda$. It follows from Theorem 9] that $(u, \lambda) \in \mathcal{S}(f)$. On the other hand, the convergences (23) entail that $(u, \lambda) \in \mathcal{S}(f) \cap C$, due to the weak closedness of the set $C$. Therefore, we conclude that $\mathcal{S}$ is a strongly-weakly upper semicontinuous mapping.

Finally, since $\mathcal{S}$ is a strongly-weakly u.s.c. mapping with weakly closed values, we are now in a position to apply Theorem 4 to obtain the desired result that $\mathcal{S}$ has a strongly-weakly closed graph. This completes the proof.

REMARK 12. From the proofs of Theorems 10 and 11, we can see that the essence of the coercivity condition $H(A)$ (iii) and inequality $H(J)($ ii) is to guarantee the following condition

$$
\frac{\langle A u, u\rangle-J^{0}(\gamma u ;-\gamma u)}{\|u\|_{V}} \rightarrow+\infty, \quad \text { as } \quad\|u\|_{V} \rightarrow \infty .
$$

Moreover, it can be observed that if $h$ is coercive in the following sense

$$
\frac{h(u)}{\|u\|_{V}} \rightarrow+\infty, \quad \text { as }\|u\|_{V} \rightarrow \infty
$$

and hypotheses $H(J)(i), H(J)($ iii), and $H(A)($ ii) hold, then condition 24) is automatically satisfied. In that case, assumptions $H(J)($ ii) and $H(A)($ iii) could be removed.

Lemma 13. Assume that $H(J)(i), H(J)($ iii), and $H(A)$ (ii) are fulfilled. If the function $h$ is coercive in the sense of (25), then condition (24) holds.

Proof. Let $u \in V$. By the $h$-relaxed monotonicity of $A \cdot+\gamma^{*} \partial J(\gamma \cdot)$, we deduce

$$
\begin{array}{r}
h(u) \leq\left\langle A u-A 0_{V}, u\right\rangle+\left\langle\xi_{u}-\xi_{0}, \gamma u\right\rangle_{X^{*} \times X}\left(\text { for all } \xi_{0} \in \partial J\left(0_{X}\right)\right) \\
\leq\langle A u, u\rangle+\left(\left\|A 0_{V}\right\|_{V^{*}}+\left\|\gamma^{*} \partial J\left(0_{X}\right)\right\|_{V^{*}}\right)\|u\|_{V}-J^{0}(\gamma u ;-\gamma u),
\end{array}
$$

where $\xi_{u} \in \partial J(\gamma u)$ is such that $\left\langle\xi_{u},-\gamma u\right\rangle_{X^{*} \times X}=J^{0}(\gamma u ;-\gamma u)$. This implies

$$
\frac{h(u)}{\|u\|_{V}}-\left(\left\|A 0_{V}\right\|_{V^{*}}+\left\|\gamma^{*} \partial J\left(0_{X}\right)\right\|_{V^{*}}\right) \leq \frac{\langle A u, u\rangle-J^{0}(\gamma u ;-\gamma u)}{\|u\|_{V}} .
$$

Now, by the coercivity condition (25) and hypothesis $H(J)($ iii) we obtain the desired conclusion. 
REMARK 14. Note that if $A: V \rightarrow V^{*}$ is strongly monotone with constant $m_{A}>0$ and $\partial J: X \rightarrow X^{*}$ is relaxed monotone with constant $m_{J} \geq 0$, i.e.,

$$
\langle A u-A v, u-v\rangle \geq m_{A}\|u-v\|_{V}^{2} \quad \text { and } \quad\left\langle\xi_{w}-\xi_{x}, w-x\right\rangle_{X^{*} \times X} \geq-m_{J}\|w-x\|_{X}^{2}
$$

for all $u, v \in V$, all $x, w \in X$, and all $\xi_{w} \in \partial J(w), \xi_{x} \in \partial J(x)$, and the inequality $m_{J}\|\gamma\|_{\mathcal{L}(V, X)}^{2}<m_{A}$ holds, then $h: V \rightarrow \mathbb{R}$ chosen as $h(u)=\left(m_{A}-m_{J}\|\gamma\|_{\mathcal{L}(V, X)}^{2}\right)\|u\|_{V}^{2}$ satisfies condition (24).

In what follows, we introduce a multivalued function $\mathcal{S}_{1}: V^{*} \rightarrow 2^{V}$ defined by

$$
\mathcal{S}_{1}(f):=\{u \in V \mid \text { there exists } \lambda \in \Lambda \text { such that }(u, \lambda) \in \mathcal{S}(f)\} .
$$

The unique solvability is of fundamental importance in numerical analysis of the problem. So, this brings about the natural question of whether the mixed variationalhemivariational inequality has a unique solution. The following theorem examines a significant conclusion that Problem 1 has at least a solution $(u, \lambda) \in V \times \Lambda$, which is unique in its first component.

TheOREM 15. Let $\Lambda$ be a nonempty, closed and convex subset of $E$ with $0_{E} \in \Lambda$. If hypotheses $H(A), H(b), H(J), H(h)$, and $H(\gamma)$ are fulfilled, then Problem 1 has at least one solution $(u, \lambda) \in V \times \Lambda$, which is unique in its first component.

Proof. The existence of solution is a direct consequence of Theorem 10. Now, we shall prove the uniqueness in the first component of the pair solution for Problem 1 . Let $\left(u_{1}, \lambda_{1}\right) \in V \times \Lambda$ and $\left(u_{2}, \lambda_{2}\right) \in V \times \Lambda$ be solutions to Problem 1, so, we have

$$
\begin{aligned}
& \left\langle A\left(u_{i}\right), v-u_{i}\right\rangle+b\left(v-u_{i}, \lambda_{i}\right)+J^{0}\left(\gamma u_{i} ; \gamma v-\gamma u_{i}\right) \geq\left\langle f, v-u_{i}\right\rangle \text { for all } v \in V, \\
& b\left(u_{i}, \rho-\lambda_{i}\right) \leq 0 \quad \text { for all } \rho \in \Lambda
\end{aligned}
$$

with $i=1,2$. We take $\rho=\lambda_{2}$ and $\rho=\lambda_{1}$ in (27) for $i=1$ and 2, respectively, then, we sum up the resulting inequalities to obtain

$$
b\left(u_{1}-u_{2}, \lambda_{2}-\lambda_{1}\right)=b\left(u_{1}, \lambda_{2}-\lambda_{1}\right)+b\left(u_{2}, \lambda_{1}-\lambda_{2}\right) \leq 0 .
$$

On the other hand, inserting $v=u_{2}$ in (26) for $i=1$ and $v=u_{1}$ in (26) for $i=2$, accordingly, and combining the resulting inequalities with (28), we can find elements $\xi_{u_{1}} \in \partial J\left(\gamma u_{1}\right)$ and $\xi_{u_{2}} \in \partial J\left(\gamma u_{2}\right)$ such that

$$
\left\{\begin{array}{l}
J^{0}\left(\gamma u_{1} ; \gamma u_{2}-\gamma u_{1}\right)=\left\langle\xi_{u_{1}}, \gamma u_{2}-\gamma u_{1}\right\rangle_{X^{*} \times X}, \\
J^{0}\left(\gamma u_{2} ; \gamma u_{1}-\gamma u_{2}\right)=\left\langle\xi_{u_{2}}, \gamma u_{1}-\gamma u_{2}\right\rangle_{X^{*} \times X}
\end{array}\right.
$$

and

$$
\begin{aligned}
& \left\langle A\left(u_{1}\right)-A\left(u_{2}\right), u_{1}-u_{2}\right\rangle-\left(\left\langle\xi_{u_{1}}, \gamma u_{2}-\gamma u_{1}\right\rangle_{X^{*} \times X}+\left\langle\xi_{u_{2}}, \gamma u_{1}-\gamma u_{2}\right\rangle_{X^{*} \times X}\right) \\
& \quad=\left\langle A\left(u_{1}\right)-A\left(u_{2}\right), u_{1}-u_{2}\right\rangle-\left(J^{0}\left(\gamma u_{1} ; \gamma u_{2}-\gamma u_{1}\right)+J^{0}\left(\gamma u_{2} ; \gamma u_{1}-\gamma u_{2}\right)\right) \\
& \quad \leq b\left(u_{2}-u_{1}, \lambda_{1}\right)+b\left(u_{1}-u_{2}, \lambda_{2}\right)=b\left(u_{1}-u_{2}, \lambda_{2}-\lambda_{1}\right) \leq 0 .
\end{aligned}
$$


The latter combined with the $h$-relaxed monotonicity of $A \cdot+\gamma^{*} \partial J(\gamma \cdot)$ implies

$$
h\left(u_{1}-u_{2}\right) \leq\left\langle A\left(u_{1}\right)+\gamma^{*} \xi_{u_{1}}-A\left(u_{2}\right)-\gamma^{*} \xi_{u_{2}}, u_{1}-u_{2}\right\rangle \leq 0 .
$$

Recalling that $h(v)>0$ for all $v \in V \backslash\left\{0_{V}\right\}$, we conclude that $u_{1}=u_{2}$.

In what follows, when the first component of the pair solution to Problem 1 is unique, we have the following stability result.

TheOREM 16. Let $\Lambda$ be a nonempty, closed and convex subset of $E$ with $0_{E} \in \Lambda$. If hypotheses $H(A), H(b), H(J), H(h)$, and $H(\gamma)$ hold, then the mapping $\mathcal{S}_{1}: V^{*} \rightarrow V$ is weakly continuous, i.e., $f_{n} \rightarrow f$ in $V^{*}$ implies $\mathcal{S}_{1}\left(f_{n}\right) \rightarrow \mathcal{S}_{1}(f)$ weakly in $V$. Moreover, if there exist $\tau>1$ and $c_{h}>0$ such that $h(u) \geq c_{h}\|u\|_{V}^{\tau}$ for all $u \in V$, then we have

$$
\left\|\mathcal{S}_{1}\left(f_{1}\right)-\mathcal{S}_{1}\left(f_{2}\right)\right\|_{V} \leq c_{h}^{\frac{1}{\tau-1}}\left\|f_{1}-f_{2}\right\|_{V^{*}}^{\frac{1}{\tau-1}}
$$

Proof. Let $\left\{f_{n}\right\} \subset V^{*}$ be a sequence such that $f_{n} \rightarrow f$ in $V^{*}$. Let $\left(u_{n}, \lambda_{n}\right) \in V \times \Lambda$ be a solution to Problem 1 corresponding to $f_{n}$. It follows from the assertion (iii) of Theorem 11 that the sequence $\left\{\left(u_{n}, \lambda_{n}\right)\right\}$ is bounded in $V \times \Lambda$. The reflexivity of $V \times E$ ensures that there exist a subsequence of $\left\{\left(u_{n}, \lambda_{n}\right)\right\}$, still denoted by the same symbol, and a pair of elements $(u, \lambda) \in V \times \Lambda$ such that

$$
u_{n} \rightarrow u \text { weakly in } V \text {, and } \lambda_{n} \rightarrow \lambda \text { weakly in } E \text {, as } n \rightarrow \infty \text {. }
$$

We now claim that $(u, \lambda)$ is also a solution of Problem 1 associated with $f$. Indeed, for each $n \in \mathbb{N}$, we use Theorem 9 to get

$$
\left\langle A\left(u_{n}\right), v-u_{n}\right\rangle+b\left(v, \lambda_{n}\right)-b\left(u_{n}, \rho\right)+J^{0}\left(\gamma u_{n} ; \gamma v-\gamma u_{n}\right) \geq\left\langle f_{n}, v-u_{n}\right\rangle
$$

for all $v \in V$ and $\rho \in \Lambda$. From the $h$-relaxed monotonicity of $A \cdot+\gamma^{*} \partial J(\gamma \cdot)$, we have

$$
\left\langle A(v), v-u_{n}\right\rangle+b\left(v, \lambda_{n}\right)-b\left(u_{n}, \rho\right)+\left\langle\xi_{v}, \gamma\left(v-u_{n}\right)\right\rangle_{X^{*} \times X} \geq\left\langle f_{n}, v-u_{n}\right\rangle
$$

for all $\xi_{v} \in \partial J(\gamma v)$ and all $(v, \rho) \in V \times \Lambda$. Passing to the upper limit in (31), as $n \rightarrow \infty$, and applying Theorem 9, we conclude that $(u, \lambda)$ is a solution to Problem 1 with respect to $f$. Note that the first component of the pair solution for Problem 1 is unique, this confesses that every subsequence of $\left\{u_{n}\right\}$ converges weakly to the same limit $u$, so, we deduce that the whole sequence $\left\{u_{n}\right\}$ converges weakly to $u$ in $V$, thus is, $\mathcal{S}_{1}\left(f_{n}\right) \rightarrow \mathcal{S}(f)$ weakly in $V$, as $n \rightarrow \infty$.

Furthermore, we assume that there exist $\tau>1$ and $c_{h}>0$ such that $h(u) \geq$ $c_{h}\|u\|_{V}^{\tau}$ for all $u \in V$. Let $\left(u_{i}, \lambda_{i}\right)$ be a solution to Problem 1 corresponding to $f_{i}$, for $i=1,2$, respectively. So, we have $u_{i}=\mathcal{S}_{1}\left(f_{i}\right)$ for $i=1,2$. By an easy calculation, it turns out that

$$
\begin{aligned}
& \left\langle A\left(\mathcal{S}_{1}\left(f_{1}\right)\right)-A\left(\mathcal{S}_{1}\left(f_{2}\right)\right), \mathcal{S}_{1}\left(f_{1}\right)-\mathcal{S}_{1}\left(f_{2}\right)\right\rangle-\left(J^{0}\left(\gamma \mathcal{S}_{1}\left(f_{1}\right) ; \gamma \mathcal{S}_{1}\left(f_{2}\right)-\gamma \mathcal{S}_{1}\left(f_{1}\right)\right)\right. \\
& \left.+J^{0}\left(\gamma \mathcal{S}_{1}\left(f_{2}\right) ; \gamma \mathcal{S}_{1}\left(f_{1}\right)-\gamma \mathcal{S}_{1}\left(f_{2}\right)\right)\right) \leq\left\langle f_{1}-f_{2}, \mathcal{S}_{1}\left(f_{1}\right)-\mathcal{S}_{1}\left(f_{2}\right)\right\rangle
\end{aligned}
$$


Next, $H(A)($ ii) implies

$$
\begin{aligned}
& c_{h}\left\|\mathcal{S}_{1}\left(f_{1}\right)-\mathcal{S}_{1}\left(f_{2}\right)\right\|_{V}^{\tau} \leq h\left(\mathcal{S}_{1}\left(f_{1}\right)-\mathcal{S}_{1}\left(f_{2}\right)\right) \leq\left\langle f_{1}-f_{2}, \mathcal{S}_{1}\left(f_{1}\right)-\mathcal{S}_{1}\left(f_{2}\right)\right\rangle \\
& \quad \leq\left\|f_{1}-f_{2}\right\|_{V^{*}}\left\|\mathcal{S}_{1}\left(f_{1}\right)-\mathcal{S}_{1}\left(f_{2}\right)\right\|_{V} .
\end{aligned}
$$

Consequently, we can readily derive the inequality (29). This completes the proof.

From Theorems 10 and 11, we have the following result.

Corollary 17. Let $\Lambda$ be a nonempty, closed and convex subset of $E$ with $0_{E} \in \Lambda$. If hypotheses $H(A), H(b), H(J)$, and $H(\gamma)$ are satisfied with $h(u)=0$ for all $u \in V$, then we have

(i) the solution set $S(A, J, b, f)$ to Problem 1 is nonempty, bounded, weakly closed, and convex in $V \times \Lambda$,

(ii) the multivalued mapping $\mathcal{S}: V^{*} \rightarrow 2^{V \times \Lambda}$ defined by $f \mapsto \mathcal{S}(f):=S(A, J, b, f)$ is bounded, i.e., $\mathcal{S}$ maps bounded subsets of $V^{*}$ to bounded subsets of $V \times \Lambda$,

(iii) the multivalued mapping $f \mapsto \mathcal{S}(f)$ is strongly-weakly upper semicontinuous, (i.e., it is upper semicontinuous from $V^{*}$ endowed with the norm topology to the subsets of $V \times \Lambda$ endowed with the weak topology), and it has a strongly-weakly closed graph.

Finally, invoking Remark 12, Lemma 13, Theorems 11, 15 and 16, we obtain the following conclusion.

Corollary 18. Let $\Lambda$ be a nonempty, closed and convex subset of $E$ with $0_{E} \in \Lambda$. If hypotheses $H(A)(i)$-(ii), $H(A)(i v), H(b), H(J)(i), H(h)(i i), H(J)($ iii) and $H(\gamma)$ are fulfilled with $h(u) \geq c_{h}\|u\|^{q}$ for all $u \in V$ and some $c_{h}>0, q>1$, then Problem 1 has at least one solution $(u, \lambda) \in V \times \Lambda$, which is unique in its first component. Moreover, the following inequality holds

$$
\left\|\mathcal{S}_{1}\left(f_{1}\right)-\mathcal{S}_{1}\left(f_{2}\right)\right\|_{V} \leq c_{h}^{\frac{1}{q-1}}\left\|f_{1}-f_{2}\right\|_{V^{*}}^{\frac{1}{q-1}} \text { for all } f_{1}, f_{2} \in V^{*} .
$$

\section{References}

[1] M. Barboteu, K. Bartosz, W. Han, T. Janiczko, Numerical analysis of a hyperbolic hemivariational inequality arising in dynamic contact, SIAM J. Numer. Anal. 53 (2015), 527-550.

[2] K. Bartosz, M. Sofonea, The Rothe method for variational-hemivariational inequalities with applications to contact mechanics, SIAM J. Math. Anal. 48 (2016), 861-883.

[3] S. Carl, D. Motreanu, Extremal solutions of quasilinear parabolic inclusions with generalized Clarke's gradient, J. Differential Equations 191 (2003), 206-233.

[4] F.H. Clarke, Optimization and Nonsmooth Analysis, Wiley, Interscience, New York, 1983. 
[5] M.C. Cojocaru, A. Matei, Well-posedness for a class of frictional contact models via mixed variational formulations, Nonlinear Anal. 47 (2019), 127-141.

[6] N. Costea, V. Rădulescu, Inequality problems of quasi-hemivariational type involving set-valued operators and a nonlinear term, J. Global Optim. 52 (2012), $743-756$.

[7] Z. Denkowski, S. Migórski, N.S. Papageorgiou, An Introduction to Nonlinear Analysis: Theory, Kluwer Academic/Plenum Publishers, Boston, Dordrecht, London, New York, 2003.

[8] Z. Denkowski, S. Migórski, N.S. Papageorgiou, An Introduction to Nonlinear Analysis: Applications, Kluwer Academic/Plenum Publishers, Boston, Dordrecht, London, New York, 2003.

[9] K. Fan, Some properties of convex sets related to fixed point theorems, Math. Ann. 266 (1984), 519-537.

[10] W. Han, B. Reddy, On the finite element method for mixed variational inequalities arising in elastoplasticity, SIAM J. Numer. Anal. 32 (1995), 1778-1807.

[11] W. Han, M. Sofonea, M. Barboteu, Numerical analysis of elliptic hemivariational inequalities, SIAM J. Numer. Anal. 55 (2017), 640-663.

[12] W. Han, M. Sofonea, D. Danan, Numerical analysis of stationary variational-hemivariational inequalities, Numer. Math. 139 (2018), 563-592.

[13] A. Matei, A variational approach via bipotentials for a class of frictional contact problems, Acta Appl. Math. 134 (2014), 45-59.

[14] A. Matei, An existence result for a mixed variational problem arising from Contact Mechanics, Nonlinear Anal. 20 (2014), 74-81.

[15] A. Matei, An evolutionary mixed variational problem arising from frictional contact mechanics, Math. Mech. Solids 19 (2014), 225-241.

[16] A. Matei, Two abstract mixed variational problems and aplications in contact mechanics, Nonlinear Anal. 22 (2015), 592-603.

[17] A. Matei, A mixed hemivariational-variational problem and applications, Comput. Math. Appl. (2018), doi: 10.1016/j.camwa.2018.08.068.

[18] A. Matei, R. Ciurcea, Contact problems for nonlinearly elastic materials: weak solvability involving dual Lagrange multipliers, The ANZIAM Journal 52 (2010), 160-178.

[19] A. Matei, S. Sitzmann, K. Willner, B.I. Wohlmuth, A mixed variational formulation for a class of contact problems in viscoelasticity, Appl. Anal. 97 (2018), 1340-1356. 
[20] A. Matei, M. Sofonea, A mixed variational formulation for a piezoelectric frictional contact problem, IMA J. Appl. Math. 82 (2016), 334-354.

[21] S. Migórski, A. Ochal, Quasi-static hemivariational inequality via vanishing acceleration approach, SIAM J. Math. Anal. 41 (2009), 1415-1435.

[22] S. Migórski, A. Ochal, M. Sofonea, Nonlinear Inclusions and Hemivariational Inequalities. Models and Analysis of Contact Problems, Advances in Mechanics and Mathematics 26, Springer, New York, 2013.

[23] S. Migórski, S.D. Zeng, Hyperbolic hemivariational inequalities controlled by evolution equations with application to adhesive contact model, Nonlinear Anal. 43 (2018), 121-143.

[24] S. Migórski, S.D. Zeng, Penalty and regularization method for variationalhemivariational inequalities with application to frictional contact, Z. Angew. Math. Mech. 98 (2018), 1503-1520.

[25] S. Migórski, S.D. Zeng, A class of differential hemivariational inequalities in Banach spaces, J. Global Optim. 72 (2018), 761-779.

[26] D. Motreanu, P.D. Panagiotopoulos, Minimax Theorems and Qualitative Properties of the Solutions of Hemivariational Inequalities, Nonconvex Optim. Appl., vol. 29, Kluwer, Dordrecht, 1998.

[27] P.D. Panagiotopoulos, Nonconvex energy functions, hemivariational inequalities and substationary principles, Acta Mech. 42 (1983), 160-183.

[28] P.D. Panagiotopoulos, Inequality Problems in Mechanics and Applications, Birkhäuser, Boston, 1985.

[29] P.D. Panagiotopoulos, Hemivariational Inequalities, Applications in Mechanics and Engineering, Springer-Verlag, Berlin, 1993.

[30] M. Sofonea, A. Matei, Vatiational Inequalities with Applications. A study of Antiplane Frictional Contact Problems, Advances in Mechanics and Mathematics, vol. 18, Springer, New York, 2009.

[31] M. Sofonea, A. Matei, Mathematical Models in Contact Mechanics, London Mathematical Society Lecture Note Series 398, Cambridge University Press, 2012.

[32] M. Sofonea, A. Matei, History-dependent mixed variational problems in contact mechanics, J. Global Optim. 61 (2015), 591-614.

[33] M. Sofonea, S. Migórski, Variational-Hemivariational Inequalities with Applications, Chapman \& Hall/CRC, Monographs and Research Notes in Mathematics, Boca Raton, 2017.

[34] G.J. Tang, N.J. Huang, Existence theorems of the variational-hemivariational inequalities, J. Global Optim. 56 (2013), 605-622. 
[35] Y.B. Xiao, X.M. Yang, N.J. Huang, Some equivalence results for well-posedness of hemivariational inequalities, J. Global Optim. 61 (2015), 789-802.

[36] E. Zeidler, Nonlinear Functional Analysis and Applications II A/B, Springer, New York, 1990. 